\title{
EMPLOI DE LA THROMBÉLASTOGRAPHIE POUR L'ÉTUDE DE LA COAGULATION DU LAIT
}

\author{
par \\ Mile H. MARÇAIS
}

\section{Introduction}

La coagulation du sang, sous l'action de la prothrombine dégradée en thrombine, aussi bien que la coagulation du lait sous l'action d'enzymes dites présures (animales, végétales, bactériennes, peut-être à tort pour certaines) se traduisent par la formation d'un coagulum.

L'exploration du phénomène de la coagulation sanguine a été étudiée par divers auteurs et, en particulier, par le docteur M. Leroux [6] (1957), grâce au thrombélastographe de Hartert. Pour la secrétion de la mamelle, une note initiale fut publiée en 1964 par M. le professeur Jacquet et nous-même [4].

\section{Principe}

Les premiers auteurs ont utilisé les propriétés dynamiques apparaissant dans le milieu, lors de la formation du caillot de fibrine, mettant ainsi en évidence la transformation de la structure du milieu sanguin, puis l'évolution de cette structure dans le temps.

De la même façon, le thrombélastographe doit pouvoir permettre d'étudier le comportement de laits auxquels on ajoute de la présure. Cette étude fait l'objet de ce travail.

\section{Description rapide du thrombélastographe}

Le thrombélastographe a été très bien décrit par M. Leroux dans son remarquable ouvrage sur la thrombélastographie, auquel nous renvoyons les lecteurs qui désireraient plus de détails.

Cet appareil comporte :

1. Un système dynamique se composant de trois cuves en acier de $0,40 \mathrm{~cm}^{3}$ de capacité chacune. Ces cuves sont animées d'un mouvement de rotation partiel, très lent, périodique.

Dans chaque cuve plonge un cylindre suspendu à un fil de torsion supportant un miroir.

$2^{\circ}$ Un système lumineux permettant l'enregistrement sur papier photo-sensible des oscillations des trois miroirs correspondant 
à chacune des cuves, les cylindres étant entraînés par le mouvement de rotation des cuves lorsque le liquide qui s'y trouve, coagule.

\section{Description du diagramme obtenu sur le document photo- graphique}

Les diagrammes obtenus grâce au thrombélastographe présentent, en début d'opération, un tracé rectiligne dont l'épaisseur de trait est inférieur à $1 \mathrm{~mm}$ pendant toute la durée de pré-coagulation. Chaque minute est représentée par $2 \mathrm{~mm}$ de tracé. La longueur de cet axe dépend de la quantité de présure ajoutée au lait, de la force de cette présure et de la qualité du lait étudié.

Dès la prise en masse du liquide, le tracé épaissit puis se dédouble en deux branches qui s'écartent plus ou moins rapidement suivant la quantité de présure agissant sur le lait.

L'ouverture maximale des branches du diagramme dépend, entre autres variables, de l'élasticité du caillot. Y interviennerıt la qualité du lait, sa teneur en sels minéraux. En tout cas, on constate que le coagulum formé est beaucoup moins élastique et moins rétractile que celui du sang (image $\mathrm{N}$ de la figure 2). Il pourrait être, comme on le fait en hématologie, appelé adynamique et se rapprocher de ce que l'on obtient avec le sang privé de plaquettes par centrifugation à froid.

\section{Détails techniques}

Le thrombélastographe de Hartert fonctionne à $37^{\circ} \mathrm{C}$. Nous avons employé, pendant cette étude, du lait frais entier provenant de mélanges de plusieurs laits et de la présure Boll diluée au 1/100e et titrant tantôt 8000 , tantôt 5500 unités à $37^{\circ} \mathrm{C}$.

Après chaque addition de présure, nous avons aussitôt déclanché un chronomètre, afin de connaître le laps de temps écoulé entre l'emprésurage et la mise en marche du système d'enregistrement photographique.

\section{Remarques}

$1^{\circ}$ Il est difficile de mesurer des temps de coagulation supérieurs à 30 minutes. Il se forme, en effet, de la crème à la surfacts des cuves. Cette pellicule entraîne le cylindre et fausse les mesures, On doit, pour pallier cet inconvénient, surveiller le spot lu.nineux se déplaçant sur l'échelle graduée du thrombélastographe et: lors de déplacements parasites, retirer la crème sans toucher au cylindre ni l'entraîner.

20 Il est important de mélanger soigneusement la présure au lait pour que, lors du prélèvement et du remplissage des cuves, la solution soit bien homogène. 
$3^{\circ}$ Le papier photographique doit être enroulé convenablement sur le tambour aprês chaque sortie, le début de l'enregistrement risquant d'être fait sur une partie ayant vu le jour. Il faut, également, vérifier que le système d'enroulement du papier fonctionne bien.

\section{Etude du temps de coagulation du lait sous l'action de la présure diluée au 1/100e}

Nous avons opéré sur des échantillons de lait de $20 \mathrm{~cm}^{3}$ auxquels nous avons ajouté des volumes décroissants de présure, titrant 8000 unités à $37^{\circ} \mathrm{C}$, et diluée au $1 / 100^{\circ}$.

Le temps de coagulation est inversement proportionnel à la quantité de présure ajoutée. Les courbes obtenues présentent une forte pente pour des quantités de présure supérieures à $0,75 \mathrm{~cm}^{3}$ de présure et deviennent presque horizontales pour des quantités de présure supérieures à $1,75 \mathrm{~cm}^{3}$. Le temps de coagulation dépend alors de la qualité du lait considéré (fig. 1).

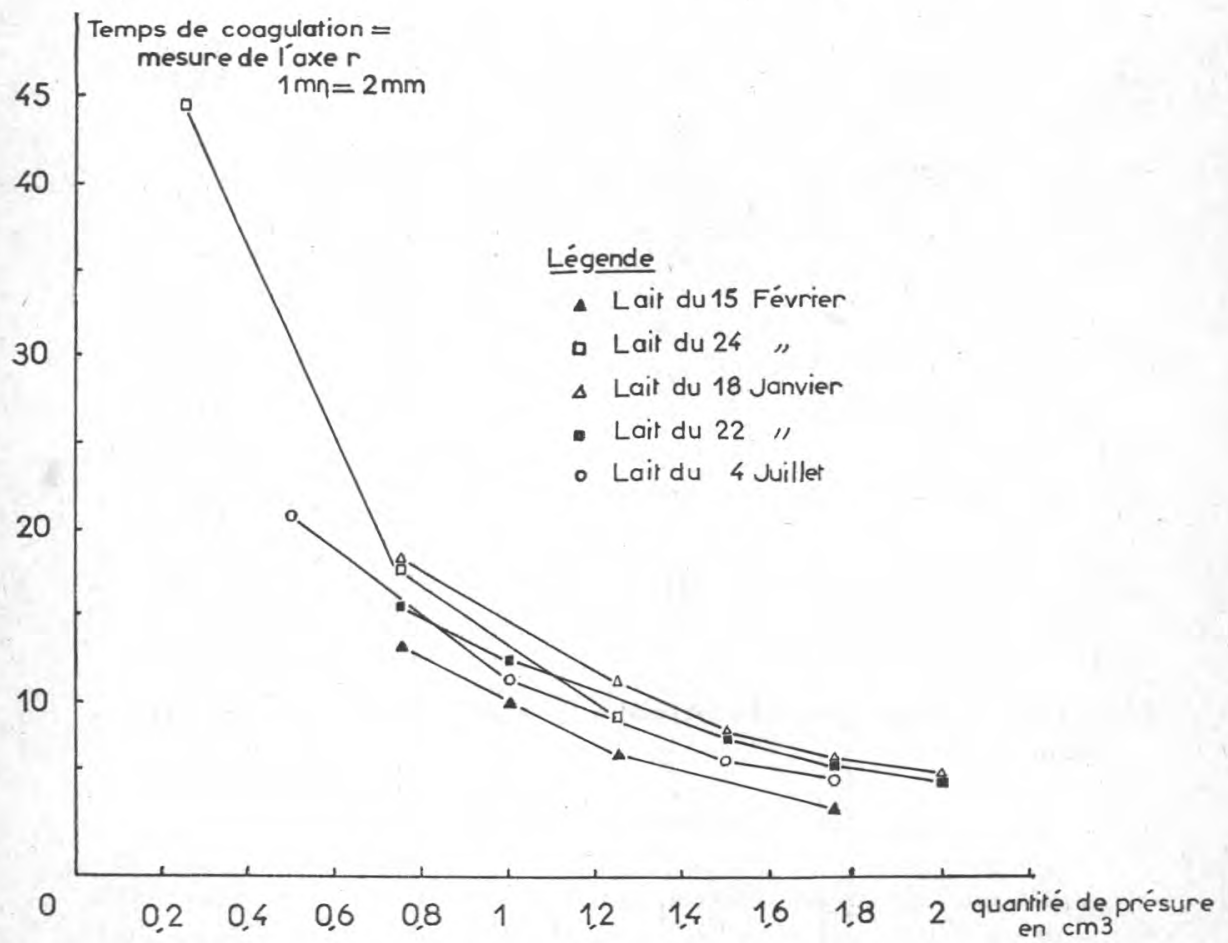

Fig. 1. - Mesure du temps de coagulation du lait d'après la longueur de l'axe horizontal $r$ du thrombélastogramme- Influence de l'activité de la présure. (Lait cru entier + présure Boll $1 / 100^{\mathrm{e}}$ ). 


\section{TABLEAU I}

Résultats obtenus aved la présure boll au $1 / 100^{e}$

\begin{tabular}{|c|c|c|c|c|}
\hline Laits crus entiers & $\begin{array}{l}\text { Volume } \\
\qquad(m\end{array}$ & de lait & $\begin{array}{c}\text { Volume } \\
\text { de présure } \\
\text { diluée au } 1 / 100^{\circ} \\
(\mathrm{ml})\end{array}$ & $\begin{array}{l}\text { Temps de } \\
\text { coagulation } \\
\left(\begin{array}{ll}m n & s\end{array}\right)\end{array}$ \\
\hline Lait du 18 janvier . . & $\begin{array}{l}\mathrm{P} \\
\mathrm{P}^{\prime}, \\
\text { O', } \\
\mathrm{N}^{\prime \prime} \\
\mathrm{O}^{\prime} \\
\mathrm{N},\end{array}$ & $\begin{array}{l}20 \\
- \\
- \\
-\end{array}$ & $\begin{array}{l}2 \\
1,75 \\
1,50 \\
1,25 \\
1 \\
0,75\end{array}$ & $\begin{array}{l}6 \\
7 \\
8,30 \\
11,15 \\
15,45 \\
18\end{array}$ \\
\hline Lait du 22 janvier $\ldots \ldots \ldots$ & $\begin{array}{l}\mathrm{N} \\
\mathrm{O} \\
\mathrm{P} \\
\mathrm{N}, \\
\mathrm{O}^{\prime} \\
\mathrm{P}^{\prime}\end{array}$ & $\begin{array}{l}20 \\
- \\
- \\
-\end{array}$ & $\begin{array}{l}2 \\
1,75 \\
1,50 \\
1,25 \\
1 \\
0,75\end{array}$ & $\begin{array}{c}5,30 \\
4,30 \\
8,15 \\
6 \\
12,30 \\
15,30\end{array}$ \\
\hline Lait du 15 février & $\begin{array}{l}\mathrm{P} \\
\mathrm{O} \\
\mathrm{N} \\
\mathrm{O}\end{array}$ & $\frac{20}{-}$ & $\begin{array}{l}1,75 \\
1,25 \\
0,75 \\
1\end{array}$ & $\begin{array}{l}4 \\
6,45 \\
13,15 \\
10\end{array}$ \\
\hline Lait du 24 février & $\begin{array}{l}\mathrm{P} \\
\mathrm{O} \\
\mathrm{N}\end{array}$ & $\frac{20}{-}$ & $\begin{array}{l}1,25 \\
0,75 \\
0,25\end{array}$ & $\begin{array}{r}9,15 \\
17,47 \\
44,57\end{array}$ \\
\hline Lait du 4 juillet $\ldots$ & $\begin{array}{l}\mathrm{P} \\
\mathrm{O} \\
\mathrm{N} \\
\mathrm{O}\end{array}$ & $\frac{20}{-}$ & $\begin{array}{l}0,50 \\
1 \\
1,50 \\
1,75\end{array}$ & $\begin{array}{r}20,50 \\
11,23 \\
6,41 \\
5,48\end{array}$ \\
\hline
\end{tabular}

Etude de l'action de sels minéraux sur le temps de coagulation du lait

\section{A - Action du chlorure de calcium}

A $40 \mathrm{~cm}^{3}$ de prise d'essai, nous avons ajouté des quantités croissantes d'une solution de chlorure de calcium à 20 p. 100. Après avoir soigneusement mélangé, nous avons porté le lait à $37^{\circ}$ C. Puis, comme précédemment, nous avons additionné ce mélange de $0,75 \mathrm{~cm}^{3}$ de présure Boll au $1 / 100^{\mathrm{e}}$, puis de $1 \mathrm{~cm}^{3}$. 
a) Le temps de coagulation a été raccourci proportionnellement à la quantité de sel de calcium ajoutée, ceci jusqu'à un volume de $0,1 \mathrm{~cm}^{3}$. Au-delà de cette valeur, le raccourcissement de temps de coagulation devient très faible, presque nul.

b) Augmentation de l'élasticité du caillot: les branches des diagrammes obtenues sont beaucoup plus écartées que celles que nous avons enregistré en l'absence d'addition de chlorure calcique et leur écartement est plus rapide, rappelant l'allure des diagrammes obtenus avec du sang sous l'action de la thrombine.

Résultats obtenus après addition de ChLORURe de CALCIUM

I. Lait du 19 mars : teneur primitive en calcium exprimée par litre en $\mathrm{CaO}$ : 1,804 g par litre

II. Lait du 6 juin : teneur primitive en calcium exprimée en $\mathrm{CaO}$ : $1,312 \mathrm{~g}$ par litre

III. Lait du 11 juil. : teneur primitive en calcium : 1,148 g de CaO par litre TABLEAU II

\begin{tabular}{|c|c|c|c|c|c|}
\hline & \multicolumn{2}{|c|}{$\begin{array}{l}\text { Volume } \\
\text { de lait }\end{array}$} & $\begin{array}{c}\text { Volume } \\
\text { de chlorure } \\
\text { de calcium } \\
\text { à } 20 \text { p. } 100 \\
\quad(\mathrm{ml})\end{array}$ & $\begin{array}{c}\text { Volume } \\
\text { de présure } \\
\text { au } 1 / 100^{\mathrm{e}} \\
\\
(\mathrm{ml})\end{array}$ & $\begin{array}{c}\text { Temps de } \\
\text { coagulation } \\
(m n s)\end{array}$ \\
\hline \multirow{4}{*}{$\begin{array}{l}\text { Lait du } 19 \text { mars } \ldots \ldots \ldots \ldots \ldots \\
\text { Titre de la présure : } 5500 \quad \text { U }\end{array}$} & $\mathrm{P}$ & 40 & 0,1 & 0,75 & 12,45 \\
\hline & $\mathrm{O}$ & - & 0,4 & - & 5,55 \\
\hline & $\mathrm{O}^{\prime}$ & - & 0,5 & - & 4,45 \\
\hline & $\mathbf{N}$ & - & 0,6 & - & 2,55 \\
\hline \multirow{8}{*}{$\begin{array}{l}\text { Lait du } 6 \text { juin } \ldots \ldots \ldots \ldots \ldots \\
\text { Titre de la présure : } 5500 \mathrm{U} \text {, }\end{array}$} & 0 & 40 & 0,05 & - & 16,30 \\
\hline & $\mathbf{P}$ & - & 0,1 & - & 12,5 \\
\hline & $\mathbf{N}$ & - & 0,2 & - & 12 \\
\hline & $\mathrm{O}$ & - & 0,3 & - & 8,45 \\
\hline & $\mathrm{P}$ & & 0,4 & - & 5,45 \\
\hline & $N$ & - & 0,5 & - & 5,45 \\
\hline & O & - & 0,6 & - & 5,15 \\
\hline & $\mathbf{P}$ & - & 0,8 & - & 4,15 \\
\hline \multirow{13}{*}{$\begin{array}{l}\text { Lait du } 11 \text { juillet } \ldots \ldots \ldots \ldots \\
\text { Titre de la présure }: 8000 \mathrm{U} \text {. }\end{array}$} & $\mathrm{N}$ & 40 & 0 & 0,75 & 31,30 \\
\hline & $\mathbf{P}^{\prime \prime}$ & - & 0,05 & - & 22,15 \\
\hline & $\mathrm{O}$ & - & 0,1 & - & 9,15 \\
\hline & $\mathbf{P}$ & - & 0,2 & - & 6,15 \\
\hline & $\mathrm{P}^{\prime}$ & - & 0,3 & - & 5,15 \\
\hline & $\mathrm{O}^{\prime}$, & - & 0,4 & - & 7,45 \\
\hline & $\begin{array}{l}N^{\prime \prime} \\
O^{\prime \prime}\end{array}$ & - & $\begin{array}{l}0,5 \\
1\end{array}$ & - & 4,15 \\
\hline & $0^{3}$ & & 1 & - & 4 \\
\hline & 0 & 40 & 0,2 & 1 & 2,15 \\
\hline & $\mathrm{P}$ & - & 0,3 & - & 1,45 \\
\hline & $\mathrm{P}^{\prime}$ & - & 0,4 & - & 1,45 \\
\hline & O' & - & 0,5 & - & 1,15 \\
\hline & $\mathbf{N}^{\prime}$ & - & 1 & - & 45 \\
\hline
\end{tabular}




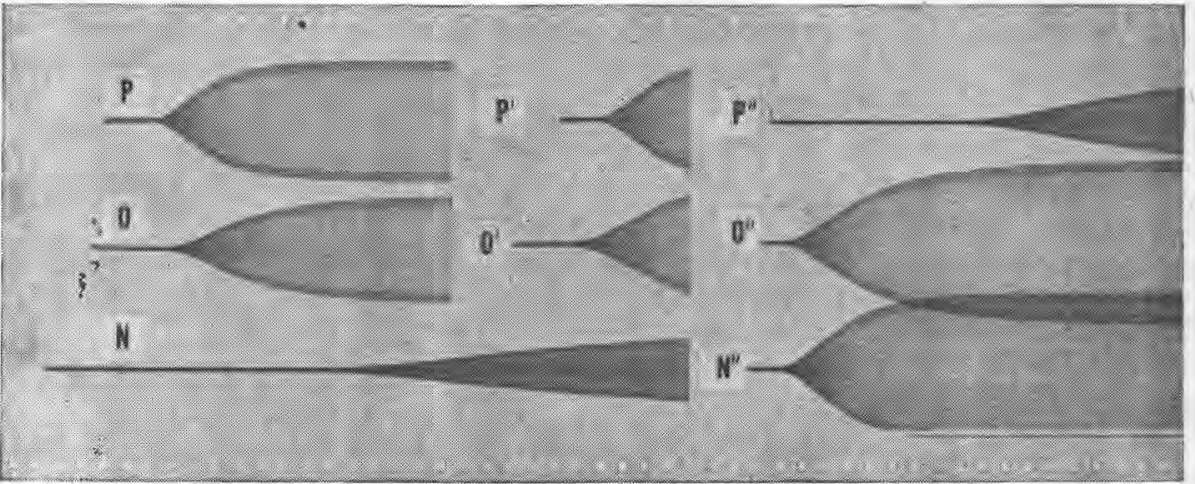

Frg. 2. - Diagrammэ représэntant la coagulation de $40 \mathrm{ml}$ de lait additionné de quantités croissantes d'une solution de chlorure de calcium a 20 p. 100. Il faut ajouter 30 secondes à chaque évaluation de la longueur de l'axe horizontal " $\mathrm{r} * \mathrm{~N}$ a été obtenu avec du lait sans chlorure de calcium. C'est 1: diagramme témoin. (Echantillon du 11 juillet 1964, tableau II).

\section{Temps de} coagulation

en $m n$

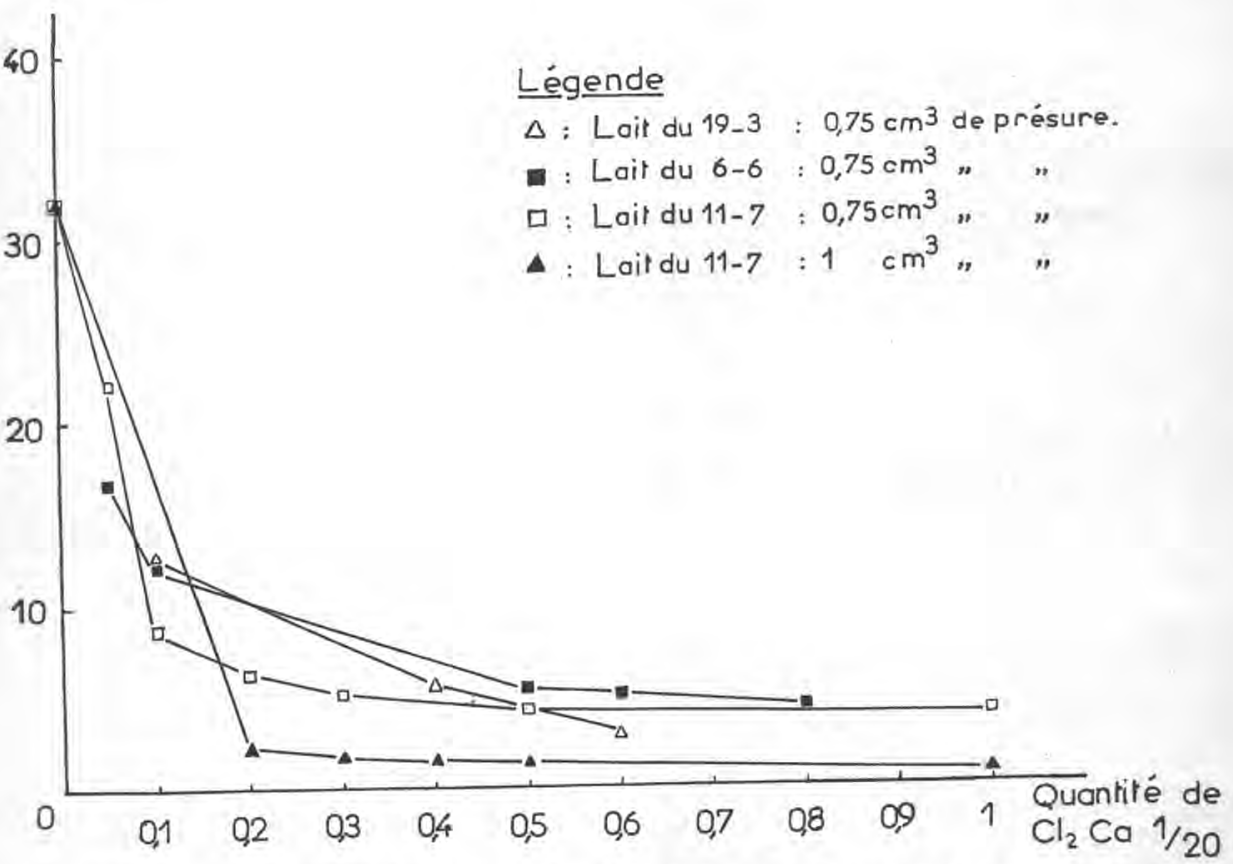

Fig. 3. - Influence de l'adjonction de calcium sur le temps de coagulation du lait par la présure. (Lait cru entier + présure $\mathrm{Boll} 1 / 100^{\mathrm{e}}+\mathrm{Cl}_{2} \mathrm{Ca}$ à 20/100). 
Nos résultats ont été traduits sur le graphique 3 .

\section{Remarques}

1) Sur la courbe (lait du 11 juillet), nous avons obtenu, pour $0,4 \mathrm{ml}$ de solution de chlorure de calcium, un temps de coagulation de $7 \mathrm{mn} 45 \mathrm{~s}$, sur la courbe (lait du 6 juin), pour $0,2 \mathrm{ml}$, un temps de $12 \mathrm{mn}$.

Ces temps trop élevés sont dus, sans doute, à une homogénéisation défectueuse du mélange de lait calcifié-présure.

2) Si l'on compare ces deux laits, le lait du 6 juin contient $1,312 \mathrm{~g}$ de calcium exprimé en $\mathrm{CaO}$ par litre, celui du 11 juillet $1,148 \mathrm{~g}$.

La courbe obtenue, pour le deuxième de ces laits, devrait donc avoir une pente supérieure à celle du lait du 6 juin, ce qui n'est pas. L'explication en est que la présure employée le 6 juin titrait 5000 unités à $37^{\circ} \mathrm{C}$, alors que celle utilisée pour le second titrait 8000 unités.

\section{Temps de coagulation en $m n$}

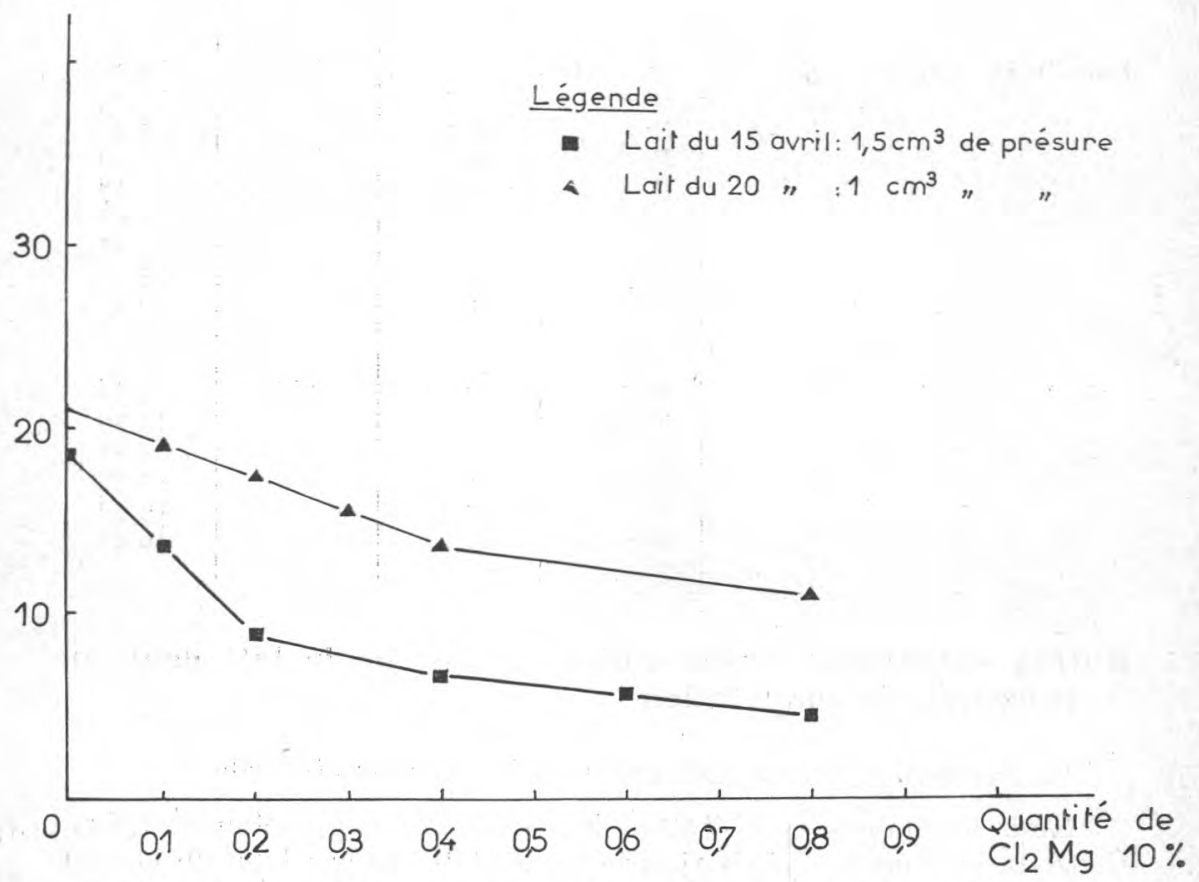

Fig. 4. - Action des ions de magnésium ajoutés au lait sur le temps de coagulation par la présure. (Lait eru entier $+\mathrm{Cl}_{2} \mathrm{Mg}$ à $10 \mathrm{p} .100+$ présure Boll 1/100 $)$. 
B - Action du chlorure de magnésium en solution à $10 p \quad p 100$

A $40 \mathrm{ml}$ de lait entier et cru, nous avons ajouté des quantité croissantes de chlorure de magnésium à 10 p. 100. (Voir fig. 4 et tableau III).

Nous avons fait coaguler ce lait en présence de $1,50 \mathrm{ml}$; puis de $0,75 \mathrm{ml}$ de présure diluée au $1 / 100^{\mathrm{e}}$. La courbe obtenue avec $1,50 \mathrm{ml}$ de présure rappelle celle donnée par le lait calcifié sous l'action de $0,75 \mathrm{ml}$ de la même enzyme. Il est à noter que pour des quantités de chlorure de magnésium supérieures de $4 \mathrm{ml}, 6 \mathrm{ml}$... la vitesse de coagulation diminue progressivement.

\section{TABLEAU III}

Résultats obtenus

\begin{tabular}{|c|c|c|c|c|c|}
\hline & $\begin{array}{l}\text { Volu } \\
\text { de lo } \\
\qquad \text { (m }\end{array}$ & & $\begin{array}{c}\text { Volume de } \\
\text { chlorure de } \\
\text { magné- } \\
\text { sium } \\
\text { d } 10 p .100 \\
(\mathrm{ml})\end{array}$ & $\begin{array}{c}\text { Volume } \\
\text { de présure } \\
\text { au } 1 / 100^{\mathrm{e}} \\
\\
(m l)\end{array}$ & $\begin{array}{l}\text { Temps } \\
\text { coagula- } \\
\quad \text { tion } \\
\\
\left(\begin{array}{ll}m n & s\end{array}\right)\end{array}$ \\
\hline Lait du 15 avril $\ldots \ldots \ldots \ldots$ & $\begin{array}{l}\mathrm{N} \\
\mathrm{O} \\
\mathrm{P} \\
\mathrm{P} \\
\mathrm{O}^{\prime} \\
\mathrm{N}^{\prime} \\
\mathrm{N}^{\prime \prime} \\
\text { O', } \\
\mathrm{P}^{\prime \prime}\end{array}$ & $\begin{array}{l}40 \\
= \\
= \\
= \\
= \\
=\end{array}$ & $\begin{array}{l}0 \\
0,1 \\
0,2 \\
0,4 \\
0,6 \\
0,8 \\
4 \\
6 \\
8\end{array}$ & $\begin{array}{c}1,50 \\
\overline{-} \\
1,50 \\
- \\
= \\
-\end{array}$ & $\begin{array}{r}18,30 \\
13,35 \\
8,45 \\
6,36 \\
6,48 \\
3,41 \\
8,07 \\
13 \\
20\end{array}$ \\
\hline Lait du 20 avril & $\begin{array}{l}\mathrm{N} \\
\mathrm{N}^{\prime} \\
\mathrm{O}^{\prime} \\
\mathrm{P}^{\prime} \\
\mathrm{O} \\
\mathrm{P}\end{array}$ & $\begin{array}{l}40 \\
- \\
- \\
-\end{array}$ & $\begin{array}{l}0 \\
0,1 \\
0,2 \\
0,3 \\
0,4 \\
0,8\end{array}$ & $\begin{array}{l}0,75 \\
= \\
= \\
-\end{array}$ & $\begin{array}{l}20,37 \\
18,36 \\
17,31 \\
15,22 \\
13,26 \\
10,45\end{array}$ \\
\hline
\end{tabular}

\section{Autres substances susceptibles d'agir sur le lait pour en provoquer la coagulation}

\section{Formation d'acide lactique par des microorganismes}

Nous avons essayé d'étudier le temps de coagulation et l'élasticité du produit formé en ajoutant à du lait $(20 \mathrm{ml}) 1 \mathrm{ml}$ de yaourt homogénéisé. Nous avons laissé incuber le mélange à l'étuve, pendant 2 heures, avant de remplir les cuves de notre thrombelastographe. 
La formation du coagulum étant très lente et le caillot étant peu élastique, les résultats obtenus sont très différents des précédents. Nous comptons reprendre plus longuement cette étude.

\section{$2{ }^{\circ}$ Acide minéral: Acide chlorhydrique}

L'addition de $0,75 \mathrm{ml}$ d'acide chlorhydrique normal à $20 \mathrm{ml}$ de lait a provoqué la coagulation immédiate du lait. Le caillot formé est élastique et nous avons pu obtenir un diagramme.

Pour $0,50 \mathrm{ml}$ et $0,25 \mathrm{ml}$ d'acide normal, la coagulation s'est faite respectivement au bout de $17 \mathrm{mn}$ et $32 \mathrm{mn}$. Mais le caillot n'était pas élastique, étant formé de fins grumeaux et le diagramme obtenu est souvent de forme irrégulière.

\section{Conclusion}

Le thrombélastographe de Hartert, grâce à son système enregistreur et aux diagrammes qu'il livre, permet d'étudier, sur document clair, les différents phénomènes de la coagulation du lait. Mais cet appareil ayant été conçu pour l'étude du sang, la formation du caillot de caséine étant beaucoup plus progressive, le point de départ du phénomène est plus difficile à situer sur l'axe horizontal $\mathbf{r}$ $\mathrm{du}$ diagramme. Nous avons arbitrairement choisi un épaississement de l'axe égal ou supérieur à $1 \mathrm{~mm}$, comme pour le sang, la largeur du trait étant de $0,5 \mathrm{~mm}$ pour un lait fluide. L'épaississement étant progressif, le début même de la coagulation nous échappe done. Il serait intéressant d'améliorer la sensibilité de cet appareil pour l'appliquer au lait avec de meilleurs résultats.

Nous avons obtenu, en employant une même présure (Présure Boll), avec chaque lait provenant pourtant d'un mélange de produits, des courbes différentes. En inversant le problème, il est possible de mesurer, à l'aide de cet appareil, la quantité de présure employée ou la force d'une présure inconnue, à condition d'avoir un lait stable, normalisé. Nous avons fait quelques mesures avec du lait Spray, mais la méthode n'est pas encore au point. Cette technique nous permettra, également, de comparer l'action, sur le lait, de la présure animale, avec celle de présures végétales ou bactériennes.

(Laboratoire de Microbiologie de la Faculté des Sciences de Caen.)

\section{Summary}

Hartert's instrument called "thrombélastographe ", designed to study the clotting of blood, can be used also to study the clotting of milk, thanks to its recording apparatus and consequenthy to its diagrams. Graphs have been obtained.

Inversely, with a stable, normalized milk, you can titrate an unknown rennet. The aforesaid method is worth testing again. 


\section{BIBLIOGRAPHIE}

[1] M. BAy. - La thrombo-élastographie. Supplément de la Revue du Praticien, 1954, $\mathrm{n}^{\circ} 6$, p. 183-185.

[2] E. Benhamoun et P. Grigner. - Le thrombélastographe et ses applications cliniques. La Presse Médicale, 1956, $\mathrm{n}^{\circ}$ 64, p. 2157-2160.

[3] H. Hartert. - Messende Verfolgung der Blut gerimmung mit dem Hellige Thrombe Elastographen nach Hartert, 1958. 1 opuscule, Hellige, Freiburg im Breisgau.

[4] J. JACQUET et H. MARĢAIs. - Enregistrement graphique du phénomène de coagulation du lait. C.R.Ac. Agric., 1964, 50, 1272-1280.

[5] J. JACQUet et R. THeVenot. - Le Lait et le Froid. Les produits laitiers et leur traitement frigorifique. Baillère, Paris, 1961. 1 volume.

[6] M. Leroux. - La thrombélastographie. Garnier éd., 1961, 1 volume.

[7] G. Marchal, M.-E. Leroux et M. SAmana. - La thrombodynamographie (thrombélastographie). Sa place actuelle en clinique, La Presse Médicale, 1960, 68, p. 1919-1922.

[8] M. Mouquin, G. Marchal, R. Sauvan, M. Samana, M. Leroux et J. Richon. - La Presse Médicale, 1958, 66, p. 1703-1704. 